\title{
Article \\ A Subclass of Multivalent Janowski Type $q$-Starlike Functions and Its Consequences
}

\author{
Qiuxia Hu ${ }^{1}$, Hari M. Srivastava ${ }^{2,3,4,5} \mathbb{D}$, Bakhtiar Ahmad ${ }^{6}\left(\mathbb{D}\right.$, Nazar Khan ${ }^{7, *}$, Muhammad Ghaffar Khan ${ }^{8} \mathbb{D}$, \\ Wali Khan Mashwani ${ }^{8}$ (D) and Bilal Khan ${ }^{9}$ (D)
}

Citation: Hu, Q.; Srivastava, H.M.; Ahmad, B.; Khan, N.; Khan, M.G.; Mashwani, W.K.; Khan, B. A Subclass of Multivalent Janowski Type $q$-Starlike Functions and Its Consequences. Symmetry 2021, 13, 1275. https://doi.org/10.3390/ sym13071275

Academic Editor: Rahmat Ellahi

Received: 19 May 2021

Accepted: 13 July 2021

Published: 16 July 2021

Publisher's Note: MDPI stays neutral with regard to jurisdictional claims in published maps and institutional affiliations.

Copyright: (C) 2021 by the authors Licensee MDPI, Basel, Switzerland. This article is an open access article distributed under the terms and conditions of the Creative Commons Attribution (CC BY) license (https:// creativecommons.org/licenses/by/ $4.0 /)$.
1 Department of Mathematics, Luoyang Normal University, Luoyang 471934, China; huqiuxia306@163.com

2 Department of Mathematics and Statistics, University of Victoria, Victoria, BC V8W 3R4, Canada; harimsri@math.uvic.ca

3 Department of Medical Research, China Medical University Hospital, China Medical University, Taichung 40402, Taiwan

4 Department of Mathematics and Informatics, Azerbaijan University, 71 Jeyhun Hajibeyli Street, Baku AZ1007, Azerbaijan

5 Section of Mathematics, International Telematic University Uninettuno, I-00186 Rome, Italy

6 Department of Mathematics, Government Degree College Mardan, Marden 23200, Pakistan; pirbakhtiarbacha@gmail.com

7 Department of Mathematics, Abbottabad University of Science and Technology, Abbottabad 22010, Pakistan

8 Institute of Numerical Sciences, Kohat University of Science and Technology, Kohat 26000, Pakistan; ghaffarkhan020@gmail.com (M.G.K.); walikhan@kust.edu.pk (W.K.M.)

9 School of Mathematical Sciences and Shanghai Key Laboratory of PMMP, East China Normal University, 500 Dongchuan Road, Shanghai 200241, China; bilalmaths789@gmail.com

* Correspondence: nazarmaths@aust.edu.pk

Abstract: In this article, by utilizing the theory of quantum (or $q$-) calculus, we define a new subclass of analytic and multivalent (or $p$-valent) functions class $\mathcal{A}_{p}$, where class $\mathcal{A}_{p}$ is invariant (or symmetric) under rotations. The well-known class of Janowski functions are used with the help of the principle of subordination between analytic functions in order to define this subclass of analytic and $p$-valent functions. This function class generalizes various other subclasses of analytic functions, not only in classical Geometric Function Theory setting, but also some $q$-analogue of analytic multivalent function classes. We study and investigate some interesting properties such as sufficiency criteria, coefficient bounds, distortion problem, growth theorem, radii of starlikeness and convexity for this newly-defined class. Other properties such as those involving convex combination are also discussed for these functions. In the concluding part of the article, we have finally given the well-demonstrated fact that the results presented in this article can be obtained for the $(\mathfrak{p}, q)$-variations, by making some straightforward simplification and will be an inconsequential exercise simply because the additional parameter $\mathfrak{p}$ is obviously unnecessary.

Keywords: analytic functions; multivalent (or $p$-valent) functions; differential subordination; $q$ derivative (or $q$-difference) operator

MSC: Primary 30C45; 30C50; 30C80; Secondary 11B65; 47B38

\section{Introduction, Definitions and Motivation}

The calculus without the notion of limits, which is known as the quantum (or q-) calculus, has influenced many scientific fields due to its important applications. The generalizations of the derivative and integral operators in $q$-calculus, which are known as the $q$-derivative and $q$-integral operators, were introduced and studied by Jackson $[1,2]$.

Recently, Anastassiu [3] and Aral [4] generalized some complex-valued operators, which are known as the $q$-Picard and $q$-Gauss-Weierstrass singular integral operators. Geometric Function Theory is no exception in this regard and many authors have already 
made a substantial contribution to the field of Complex Analysis. Ismail et al. (see [5]) presented the $q$-deformation of the familiar class $\mathcal{S}^{*}$ of starlike functions. However, in the context of Geometric Function Theory in 1989, the usage of the $q$-difference (or the $q$-derivative) operator $D_{q}$ was systematically given by Srivastava [6]. Furthermore, the survey-cum-expository review article by Srivastava [7] is potentially useful for those who are interested in Geometric Function Theory. In this review article, many various applications of the the fractional $q$-calculus, in Geometric Function Theory were systematically highlighted. Moreover, the triviality of the so-called $(\mathfrak{p}, q)$-calculus involving an obviously redundant and inconsequential additional parameter $\mathfrak{p}$ was revealed and exposed (see, for details, [7], p. 340).

Based on the aforementioned works [5,7], a number of researches got inspiration to gave and their finding to Geometric Function Theory of Complex Analysis. For example, Srivastava and Bansal [8] used the $q$-derivatives and gave close-to-convexity for certain Mittag-Leffer type functions. Kanas and Răducanu [9] defined the $q$-analogue of the Ruscheweyh derivative operator and they discussed its various important properties. The applications of this $q$-derivative operator were further studied by Mahmood and Sokół [10]. More recently, Srivastava et al. [11,12] first defined certain subclasses of $q$-starlike functions and then studied their various properties including for example some coefficient inequalities, inclusion properties, and a number of sufficient conditions. Moreover, the subclasses of $q$-starlike functions associated with the Janwoski or some other functions have been studied by the many authors (see, for example, [13-20]). For some more recent investigations based upon the $q$-calculus, we may refer the interested reader to the works in [21-38]. Our present research is a continuation of some of these earlier developments. It is fairly general in its nature as it not only generalizes many known classes, but also gives a different direction to the study of such classes.

In this article, we are essentially motivated by the recently published paper of Khan et al. in Symmetry (see [27]) and some other related works on this subject, which we have mentioned above. We first introduce a new subclass of analytic and multivalent (or $p$-valent) functions by using the concept of the $q$-calculus in association with the Janowski functions. We then study some of its geometric properties such as sufficiency criteria, coefficient bounds, radii problems, distortion theorem and growth theorem, and so on. Before stating and proving our main results, we give a brief discussion on the basics of this area which will be beneficial in understanding the work to follow.

Let $\mathcal{A}_{p}$ be the class of analytic and multivalent (or $p$-valent) functions $f(z)$ in the open unit disk

$$
\mathbb{D}=\{z: z \in \mathbb{C} \text { and }|z|<1\},
$$

with the series representation given by

$$
f(z)=z^{p}+\sum_{n=1}^{\infty} a_{n+p} z^{n+p} \quad(z \in \mathbb{D} ; p \in \mathbb{N}:=\{1,2,3, \cdots\}) .
$$

We note for $p=1$ that

$$
\mathcal{A}(1)=\mathcal{A},
$$

where $\mathcal{A}$ is the familiar class of normalized analytic functions in $\mathbb{D}$ and the class $\mathcal{A}$ is invariant (or symmetric) under rotations.

For analytic functions $f$ and $g$ in open unit disk $\mathbb{D}$, the function $f$ is said to subordinate to the function $g$ and written as

$$
f \prec g \quad \text { or } \quad f(z) \prec g(z),
$$

if there exists a Schwarz function $w$, which is analytic in $\mathbb{D}$ with

$$
w(0)=0 \quad \text { and } \quad|w(z)|<1,
$$


such that

$$
f(z)=g(w(z)) .
$$

Furthermore, if the function $g$ is univalent in $\mathbb{D}$, then it follows that

$$
f(z) \prec g(z) \quad(z \in \mathbb{D}) \Longrightarrow f(0)=g(0) \text { and } f(\mathbb{D}) \subset g(\mathbb{D}) \text {. }
$$

Definition 1. Let the $Y(z)$ is analytic in $\mathbb{D}$ with $Y(0)=1$ then $Y(z)$ said to be in the class $\mathcal{P}[A, B]$, if

$$
\mathrm{Y}(z) \prec \frac{1+A z}{1+B z} \quad(1 \leqq B<A \leqq 1) .
$$

Equivalently, we can write

$$
\left|\frac{Y(z)-1}{A-B Y(z)}\right|<1 .
$$

The class $\mathcal{P}[A, B]$ was introduced by Janowski [39].

Definition 2. Let $q \in(0,1)$ and define the $q$-number $[\lambda]_{q}$ by

$$
[\lambda]_{q}= \begin{cases}\frac{1-q^{\lambda}}{1-q} & (\lambda \in \mathbb{C}) \\ \sum_{k=0}^{n-1} q^{k}=1+q+q^{2}+\cdots+q^{n-1} & (\lambda=n \in \mathbb{N}) .\end{cases}
$$

The $q$-derivative operator $D_{q}$, also known as the $q$-difference operator, for a function $f$ is defined by

$$
D_{q} f(z)=\frac{f(z)-f(q z)}{z(1-q)} \quad(z \neq 0),
$$

where $0<q<1$. One can easily see for $n \in \mathbb{N}$ and $z \in \mathbb{D}$ that

$$
D_{q}\left\{\sum_{n=1}^{\infty} a_{n} z^{n}\right\}=\sum_{n=1}^{\infty}[n]_{q} a_{n} z^{n-1},
$$

where

$$
[n]_{q}=\frac{1-q^{n}}{1-q}=1+\sum_{l=1}^{n-1} q^{l} \quad \text { and } \quad[0]_{q}=0 .
$$

Motivated by the above-cited works in [39-44], we now define a new subclass $\mathcal{S}_{q}(p, \alpha, A, B)$ of $\mathcal{A}_{p}$ as follows.

Definition 3. A function $f \in \mathcal{A}_{p}$ is said to be in the class $\mathcal{S}_{q}(p, \alpha, A, B)$, if it satisfies the following subordination condition:

$$
\frac{1}{1-\alpha}\left(\frac{z D_{q} f(z)}{[p]_{q} f(z)}-\alpha \frac{z^{2} D_{q}^{2} f(z)}{[p]_{q}[p-1]_{q} f(z)}\right) \prec \frac{1+A z}{1+B z}
$$

or, equivalently,

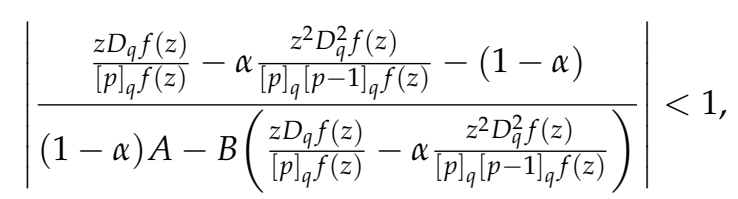

where $-1 \leqq B<A \leqq 1, \alpha \geqq 0$ and $q \in(0,1)$. 
Remark 1. First of all, it is easily seen that

$$
\lim _{q \rightarrow 1-} \mathcal{S}_{q}(1,0, A, B)=\mathcal{S}^{*}[A, B]
$$

where $\mathcal{S}^{*}[A, B]$ is the function class introduced and studied by Janowski [39]. Secondly, we have

$$
\mathcal{S}_{q}(1,0, A, B)=\mathcal{S}_{q}^{*}[A, B]
$$

where $\mathcal{S}_{q}^{*}[A, B]$ is the function class introduced and studied by Srivastava et al. [19]. Thirdly, we have

$$
\lim _{q \rightarrow 1-} \mathcal{S}_{q}(1,0,1,-1)=\mathcal{S}^{*}
$$

where $\mathcal{S}^{*}$ is the well-known class of starlike functions.

For proving our main results we will need the following lemma due to Rogosinski [45].

Lemma 1. (see [45]) Let the function $h(z)$ be given by

$$
h(z)=1+\sum_{n=1}^{\infty} d_{n} z^{n}
$$

and let another function $k(z)$ be given by

$$
k(z)=1+\sum_{n=1}^{\infty} k_{n} z^{n} .
$$

Suppose also that

$$
h(z) \prec k(z) \quad(z \in \mathbb{D}) .
$$

If $k(z)$ is univalent in $\mathbb{D}$ and $k(\mathbb{D})$ is convex, then

$$
\left|d_{n}\right| \leqq\left|k_{1}\right| \quad(n \geqq 1) .
$$

\section{The Main Results and Their Consequences}

This section is devoted to our main results. Throughout our discussion, we assume that

$$
-1 \leqq B<A \leqq 1 \quad \text { and } \quad q \in(0,1)
$$

and that

$$
\lambda_{1}=[p-1]_{q} \quad \text { and } \quad \lambda_{2}=[p+n]_{q}
$$

Theorem 1. Let $f \in \mathcal{A}_{p}$ be of the form (1). Then the function $f \in \mathcal{S}_{q}(p, \alpha, A, B)$, if and only if the following inequality holds true:

$$
\begin{aligned}
& \sum_{n=1}^{\infty}\left((1+B)\left(\lambda_{1} \lambda_{2}+\alpha \lambda_{2}[p+n-1]_{q}\right)+(1-\alpha)(1+A)[p]_{q} \lambda_{1}\right)\left|a_{n+p}\right| \\
& \quad \leqq(1-\alpha)(A-B)[p]_{q} \lambda_{1}
\end{aligned}
$$

where $\lambda_{1}$ and $\lambda_{2}$ are given in (6). 
Proof. Let us suppose that the inequality in (7) holds true. Then, in order to show that $f \in \mathcal{S}_{q}(p, \alpha, A, B)$, we only need to prove the inequality (5). For this purpose, we consider

$$
\begin{aligned}
& \left|\frac{\frac{z D_{q} f(z)}{[p]_{q} f(z)}-\alpha \frac{z^{2} D_{q}^{2} f(z)}{[p]_{q} \lambda_{1} f(z)}-(1-\alpha)}{(1-\alpha) A-B\left(\frac{z D_{q} f(z)}{[p]_{q} f(z)}-\alpha \frac{z^{2} D_{q}^{2} f(z)}{[p]_{q} \lambda_{1} f(z)}\right)}\right| \\
& \quad=\left|\frac{\lambda_{1} z D_{q} f(z)-\alpha z^{2} D_{q}^{2} f(z)-(1-\alpha)[p]_{q} \lambda_{1} f(z)}{A(1-\alpha)[p]_{q} \lambda_{1} f(z)-B\left(\lambda_{1} z D_{q} f(z)-\alpha z^{2} D_{q}^{2} f(z)\right)}\right| .
\end{aligned}
$$

Now, with the help of (1)-(3), and after some simplification, the above equation can be written as follows:

$$
\begin{aligned}
& \left|\frac{\sum_{n=1}^{\infty}\left(\lambda_{1} \lambda_{2}-\alpha \lambda_{2}[p+n-1]_{q}-(1-\alpha)[p]_{q} \lambda_{1}\right) a_{n+p} z^{n+p}}{(A-B)(1-\alpha)[p]_{q} \lambda_{1} z^{p}+\sum_{n=1}^{\infty} \Lambda_{q}\left(\lambda_{1}, \lambda_{2}\right) a_{n+p} z^{n+p}}\right| \\
& \quad=\left|\frac{\sum_{n=1}^{\infty}\left(\lambda_{1} \lambda_{2}-\alpha \lambda_{2}[p+n-1]_{q}-(1-\alpha)[p]_{q} \lambda_{1}\right) a_{n+p} z^{n}}{(A-B)(1-\alpha)[p]_{q} \lambda_{1}+\sum_{n=1}^{\infty} \Lambda_{q}\left(\lambda_{1}, \lambda_{2}\right) a_{n+p} z^{n}}\right| \\
& \quad \leqq \frac{\sum_{n=1}^{\infty}\left(\lambda_{1} \lambda_{2}+\alpha \lambda_{2}[p+n-1]_{q}+(1-\alpha)[p]_{q} \lambda_{1}\right)\left|a_{n+p}\right|}{(A-B)(1-\alpha)[p]_{q} \lambda_{1}-\sum_{n=1}^{\infty} \Lambda_{q}\left(\lambda_{1}, \lambda_{2}\right)\left|a_{n+p}\right|} \\
& \quad \leqq 1
\end{aligned}
$$

where

$$
\Lambda_{q}\left(\lambda_{1}, \lambda_{2}\right)=A(1-\alpha)[p]_{q} \lambda_{1}-B \lambda_{2}\left(\lambda_{1}-B \alpha[p+n-1]_{q}\right) .
$$

This last inequality can be rewritten as follows:

$$
\begin{aligned}
& \left|\frac{\sum_{n=1}^{\infty}\left(\lambda_{1} \lambda_{2}-\alpha \lambda_{2}[p+n-1]_{q}-(1-\alpha)[p]_{q} \lambda_{1}\right) a_{n+p} z^{n+p}}{(A-B)(1-\alpha)[p]_{q} \lambda_{1} z^{p}+\sum_{n=1}^{\infty} \Lambda_{q}\left(\lambda_{1}, \lambda_{2}\right) a_{n+p} z^{n+p}}\right| \\
& \quad=\left|\frac{\sum_{n=1}^{\infty}\left(\lambda_{1} \lambda_{2}-\alpha \lambda_{2}[p+n-1]_{q}-(1-\alpha)[p]_{q} \lambda_{1}\right) a_{n+p} z^{n}}{(A-B)(1-\alpha)[p]_{q} \lambda_{1}+\sum_{n=1}^{\infty} \Lambda_{q}\left(\lambda_{1}, \lambda_{2}\right) a_{n+p} z^{n}}\right| \\
& \quad \leqq \frac{\sum_{n=1}^{\infty}\left(\lambda_{1} \lambda_{2}+\alpha \lambda_{2}[p+n-1]_{q}+(1-\alpha)[p]_{q} \lambda_{1}\right)\left|a_{n+p}\right|}{(A-B)(1-\alpha)[p]_{q} \lambda_{1}-\sum_{n=1}^{\infty} \Lambda_{q}\left(\lambda_{1}, \lambda_{2}\right)\left|a_{n+p}\right|} \\
& \quad<1
\end{aligned}
$$

where we have used the inequality (7). This completes the direct part of the result asserted by Theorem 1 .

Conversely, let $f \in \mathcal{S}_{q}(p, \alpha, A, B)$ be given by (1). Then, from (5), we find for $z \in \mathbb{D}$ that

$$
\begin{aligned}
& \left|\frac{\frac{z D_{q} f(z)}{[p]_{q} f(z)}-\alpha \frac{z^{2} D_{q}^{2} f(z)}{[p]_{q}[p-1]_{q} f(z)}-(1-\alpha)}{(1-\alpha) A-B\left(\frac{z D_{q} f(z)}{[p]_{q} f(z)}-\alpha \frac{z^{2} D_{q}^{2} f(z)}{[p]_{q}[p-1]_{q} f(z)}\right)}\right| \\
& \quad=\left|\frac{\sum_{n=1}^{\infty}\left(\lambda_{1} \lambda_{2}-\alpha \lambda_{2}[p+n-1]_{q}-(1-\alpha)[p]_{q} \lambda_{1}\right) a_{n+p} z^{n}}{(A-B)(1-\alpha)[p]_{q} \lambda_{1}+\sum_{n=1}^{\infty} \Lambda_{q}\left(\lambda_{1}, \lambda_{2}\right) a_{n+p} z^{n}}\right| .
\end{aligned}
$$

Since

$$
\Re(z) \leqq|z|,
$$


therefore, we have

$$
\begin{aligned}
& \Re\left\{\frac{\sum_{n=1}^{\infty}\left(\lambda_{1} \lambda_{2}-\alpha \lambda_{2}[p+n-1]_{q}-(1-\alpha)[p]_{q} \lambda_{1}\right) a_{n+p} z^{n}}{(A-B)(1-\alpha)[p]_{q} \lambda_{1}+\sum_{n=1}^{\infty} \Lambda_{q}\left(\lambda_{1}, \lambda_{2}\right) a_{n+p} z^{n}}\right\} \\
& <1 \text {. }
\end{aligned}
$$

We now choose values of $z$ on the real axis in the complex $z$-plane, so that

$$
\frac{1}{1-\alpha}\left(\frac{z D_{q} f(z)}{[p]_{q} f(z)}-\alpha \frac{z^{2} D_{q}^{2} f(z)}{[p]_{q}[p-1]_{q} f(z)}\right)
$$

is real. Upon clearing the denominator in (8) and letting $z \rightarrow 1$ - through real values, we obtain (7). This completes the proof of Theorem 1.

Theorem 2. If the function $f(z)$, given by (1), belongs to the class $\mathcal{S}_{q}(p, \alpha, A, B)$, then

$$
\begin{aligned}
\left|a_{p+1}\right| \leqq & \frac{[p]_{q} \lambda_{1}(1-\alpha)(A-B)}{l(1)}, \\
\left|a_{p+2}\right| \leqq & \frac{[p]_{q} \lambda_{1}(1-\alpha)(A-B)}{l(2)}+\frac{\left([p]_{q} \lambda_{1}(1-\alpha)(A-B)\right)^{2}}{l(1) l(2)}, \\
\left|a_{p+3}\right| \leqq & \frac{[p]_{q} \lambda_{1}(1-\alpha)(A-B)}{l(3)}+\frac{\left([p]_{q} \lambda_{1}(1-\alpha)(A-B)\right)^{2}}{l(3) l(1)} \\
& +\frac{\left([p]_{q} \lambda_{1}(1-\alpha)(A-B)\right)^{2}}{l(3) l(2)}+\frac{\left([p]_{q} \lambda_{1}(1-\alpha)(A-B)\right)^{3}}{l(3) l(2) l(1)},
\end{aligned}
$$

where

$$
\lambda_{1}=[p-1]_{q}
$$

and

$$
l(n)=\lambda_{1}[p+n]_{q}-\alpha[p+n-1]_{q}[p+n]_{q}-(1-\alpha)[p]_{q} \lambda_{1} .
$$

Proof. Let $f \in \mathcal{S}_{q}(p, \alpha, A, B)$. Then

$$
\frac{1}{1-\alpha}\left(\frac{z D_{q} f(z)}{[p]_{q} f(z)}-\alpha \frac{z^{2} D_{q}^{2} f(z)}{[p]_{q} \lambda_{1} f(z)}\right)=h(z),
$$

where

$$
h(z) \prec \frac{1+A z}{1+B z}=1+(A-B) z+\cdots,
$$

is of the form given by

$$
h(z)=1+\sum_{n=1}^{\infty} d_{n} z^{n} .
$$

Thus, by the Rogosinski Lemma, we get

$$
\left|d_{n}\right| \leqq(A-B) .
$$


Now, using the series expansions of $h(z)$ and $f(z)$ in (9), together with simplification and comparsion of the coefficients of like powers of $z$, we get

$$
\begin{aligned}
& \frac{\left(\lambda_{1}-\alpha[p+n-1]_{q}\right) \lambda_{2}}{1-\alpha} a_{p+n} \\
& \quad=[p]_{q} \lambda_{1}\left(a_{p+n}+a_{p+n-1} d_{1}+a_{p+n-2} d_{2}+\ldots+a_{p+1} d_{n-1}+d_{n}\right),
\end{aligned}
$$

which can be written as follows:

$$
\begin{aligned}
& \frac{\left(\lambda_{1}-\alpha[p+n-1]_{q}\right) \lambda_{2}-(1-\alpha)[p]_{q} \lambda_{1}}{1-\alpha} a_{p+n} \\
& \quad=[p]_{q} \lambda_{1}\left(a_{p+n-1} d_{1}+a_{p+n-2} d_{2}+\ldots+a_{p+1} d_{n-1}+d_{n}\right) .
\end{aligned}
$$

Next, by first using the triangle inequality for the modulus and then applying (10), we obtain

$$
\begin{aligned}
& \frac{\left(\lambda_{1}-\alpha[p+n-1]_{q}\right) \lambda_{2}-(1-\alpha)[p]_{q} \lambda_{1}}{1-\alpha}\left|a_{p+n}\right| \\
& \leqq[p]_{q} \lambda_{1}(A-B) \sum_{i=0}^{n-1}\left|a_{p+i}\right|
\end{aligned}
$$

which implies that

$$
\left|a_{p+n}\right| \leqq \frac{(1-\alpha)[p]_{q} \lambda_{1}(A-B)}{\left(\lambda_{1}-\alpha[p+n-1]_{q}\right) \lambda_{2}-(1-\alpha)[p]_{q} \lambda_{1}} \sum_{i=0}^{n-1}\left|a_{p+i}\right| .
$$

If we now put $n=1,2$ and 3 in (11) and use the fact that $a_{p}=1$, we get the required result asserted by Theorem 2 .

Theorem 3. Let the function $f(z)$, given by (1), belong to the class $\mathcal{S}_{q}(p, \alpha, A, B)$. Then, for $|z|=r$,

$$
[p]_{q} r^{p-1}-\tau_{1} r^{p} \leqq\left|D_{q} f(z)\right| \leqq[p]_{q} r^{p-1}+\tau_{1} r^{p}
$$

where

$$
\tau_{1}=\frac{(1-\alpha)(A-B)[p+1]_{q}[p]_{q} \lambda_{1}}{(1+B)\left(\lambda_{1}+\alpha[p]_{q}\right)[p+1]_{q}+(1-\alpha)(1+A)[p]_{q} \lambda_{1}}
$$

and

$$
\lambda_{1}=[p-1]_{q}
$$

Proof. From (1) we can write

$$
D_{q} f(z)=[p]_{q} z^{p-1}+\sum_{n=1}^{\infty} \lambda_{2} a_{n+p} z^{n+p-1}
$$

so that, by applying the triangle inequality, we have

$$
\left|D_{q} f(z)\right| \leqq[p]_{q}|z|^{p-1}+\sum_{n=1}^{\infty} \lambda_{2}\left|a_{n+p}\right||z|^{n+p-1}
$$

Since $|z|=r<1$ so that $r^{n+p-1} \leqq r^{p}$, and hence we have

$$
D_{q} f(z) \leqq[p]_{q} r^{p-1}+r^{p} \sum_{n=1}^{\infty} \lambda_{2}\left|a_{n+p}\right| .
$$


Similarly, we get

$$
D_{q} f(z) \geqq[p]_{q} r^{p-1}-r^{p} \sum_{n=1}^{\infty} \lambda_{2}\left|a_{n+p}\right| .
$$

We know from (7) that

$$
\begin{aligned}
& \sum_{n=1}^{\infty}\left[(1+B)\left(\lambda_{1}+\alpha[p+n-1]_{q}\right)+\frac{(1-\alpha)(1+A) \lambda_{1}[p]_{q}}{\lambda_{2}}\right] \lambda_{2}\left|a_{n+p}\right| \\
& \quad \leqq(1-\alpha)(A-B)[p]_{q} \lambda_{1} .
\end{aligned}
$$

We also know that

$$
\begin{aligned}
& {\left[(1+B)\left(\lambda_{1}+\alpha[p]_{q}\right)+\frac{(1-\alpha)(1+A)[p]_{q} \lambda_{1}}{[p+1]_{q}}\right] \sum_{n=1}^{\infty} \lambda_{2}\left|a_{n+p}\right|} \\
& \quad \leqq \sum_{n=1}^{\infty}\left[(1+B)\left(\lambda_{1}+\alpha[p+n-1]_{q}\right)+\frac{(1-\alpha)(1+A)[p]_{q} \lambda_{1}}{\lambda_{2}}\right] \lambda_{2}\left|a_{n+p}\right|
\end{aligned}
$$

Hence, by the transitive property, we get

$$
\begin{aligned}
& {\left[(1+B)\left(\lambda_{1}+\alpha[p]_{q}\right)+\frac{(1-\alpha)(1+A)[p]_{q} \lambda_{1}}{[p+1]_{q}}\right] \sum_{n=1}^{\infty} \lambda_{2}\left|a_{n+p}\right|} \\
& \quad \leqq(1-\alpha)(A-B)[p]_{q} \lambda_{1}
\end{aligned}
$$

which implies that

$$
\sum_{n=1}^{\infty} \lambda_{2}\left|a_{n+p}\right| \leqq \frac{(1-\alpha)(A-B)[p]_{q} \lambda_{1}[p+1]_{q}}{(1+B)[p+1]_{q}\left(\lambda_{1}+\alpha[p]_{q}\right)+(1-\alpha)(1+A)[p]_{q} \lambda_{1}} .
$$

Now, by using the above inequility in (12) and (13), we obtain the required result asserted by Theorem 3 .

Theorem 4. Let the function $f(z)$, given by (1), belong to the class $\mathcal{S}_{q}(p, \alpha, A, B)$. Then, for $|z|=r$,

$$
r^{p}\left(1-\tau_{2}\right) \leqq|f(z)| \leqq r^{p}\left(1+\tau_{2}\right),
$$

where

$$
\tau_{2}=\frac{(1-\alpha)(A-B)[p]_{q} \lambda_{1}}{[p+1]_{q}(1+B)\left(\lambda_{1}+\alpha[p]_{q}\right)+(1-\alpha)(1+A)[p]_{q} \lambda_{1}} .
$$

Proof. By applying the triangle inequality in (1), and using the fact that $|z|=r$, we have

$$
|f(z)| \leqq r^{p}+\sum_{n=1}^{\infty}\left|a_{n+p}\right| r^{n+p}
$$

Since $|z|=r<1$ so that $r^{n+p}<r^{p}$, therefore, the above relation becomes

$$
|f(z)| \leqq r^{p}+r^{p} \sum_{n=1}^{\infty}\left|a_{n+p}\right| .
$$


Similarly, we get

$$
|f(z)| \geqq r^{p}-r^{p} \sum_{n=1}^{\infty}\left|a_{n+p}\right|
$$

We know from (7) that

$$
\begin{aligned}
\sum_{n=1}^{\infty}[ & \left.\lambda_{2}(1+B)\left(\lambda_{1}+\alpha[p+n-1]_{q}\right)+(1-\alpha)(1+A)[p]_{q} \lambda_{1}\right]\left|a_{n+p}\right| \\
& \leqq(1-\alpha)(A-B)[p]_{q} \lambda_{1}
\end{aligned}
$$

But

$$
\begin{aligned}
& {\left[[p+1]_{q}(1+B)\left(\lambda_{1}+\alpha[p]_{q}\right)+(1-\alpha)(1+A)[p]_{q} \lambda_{1}\right] \sum_{n=1}^{\infty}\left|a_{n+p}\right|} \\
& \quad \leqq \sum_{n=1}^{\infty}\left[\lambda_{2}(1+B)\left(\lambda_{1}+\alpha[p+n-1]_{q}\right)+(1-\alpha)(1+A)[p]_{q} \lambda_{1}\right]\left|a_{n+p}\right| \\
& \quad \leqq(1-\alpha)(A-B)[p]_{q}[p-1]_{q} .
\end{aligned}
$$

Hence

$$
\begin{aligned}
& {\left[[p+1]_{q}(1+B)\left(\lambda_{1}+\alpha[p]_{q}\right)+(1-\alpha)(1+A)[p]_{q} \lambda_{1}\right] \sum_{n=1}^{\infty}\left|a_{n+p}\right|} \\
& \quad \leqq(1-\alpha)(A-B)[p]_{q}[p-1]_{q^{\prime}}
\end{aligned}
$$

which gives

$$
\sum_{n=1}^{\infty}\left|a_{n+p}\right| \leqq \frac{(1-\alpha)(A-B)[p]_{q} \lambda_{1}}{[p+1]_{q}(1+B)\left(\lambda_{1}+\alpha[p]_{q}\right)+(1-\alpha)(1+A)[p]_{q} \lambda_{1}} .
$$

Now, by substituting from the above inequality into (14) and (15), we get the required result asserted by Theorem 4 .

Now before starting radii problems let us remaind the definition of important classes of multivalent starlike and convex functions.

A function $f \in \mathcal{A}_{p}$, is said to be multivalent starlike functions of order $\sigma$ if it satisfies the following inequality

$$
\Re\left\{\frac{z f^{\prime}(z)}{p f(z)}\right\}>\sigma, z \in \mathbb{D},(0 \leq \sigma \leq p, p \in \mathbb{N}),
$$

and we denoted this class by $\mathcal{S}_{p}^{*}(\sigma)$.

Furthermore, by $\mathcal{C}_{p}(\sigma)$ we mean the class of multivalent convex functions, that is a function $f \in \mathcal{A}_{p}$ and satisfies the inequality below

$$
\Re\left\{\frac{z\left(z f^{\prime}(z)\right)^{\prime}}{p^{2} f(z)}\right\}>\sigma, z \in \mathbb{D},(0 \leq \sigma \leq p, p \in \mathbb{N}) .
$$

Theorem 5. Let $f \in \mathcal{S}_{q}(p, \alpha, A, B)$. Then $f \in \mathcal{C}_{p}(\sigma)$ for $|z|<r_{1}$, where

$$
r_{1}=\left\{\inf \left(\frac{p(p-\sigma)\left((1+B) \lambda_{2}\left(\lambda_{1}+\alpha[p+n-1]_{q}\right)+(1-\alpha)(1+A)[p]_{q} \lambda_{1}\right)}{(p+n)(n+p-\sigma)(1-\alpha)(A-B)[p]_{q} \lambda_{1}}\right)^{\frac{1}{n}}: n \in \mathbb{N}\right\}
$$


Proof. Suppose that $f \in \mathcal{S}_{q}(p, \alpha, A, B)$. Then, in order to prove that $f \in \mathcal{C}_{p}(\sigma)$, we only need to show that

$$
\left|\frac{z f^{\prime \prime}(z)-(p-1) f^{\prime}(z)}{z f^{\prime \prime}(z)+(1-2 \sigma+p) f^{\prime}(z)}\right|<1
$$

Using (1) followed by some simplifications, we have

$$
\sum_{n=1}^{\infty} \frac{(p+n)(n+p-\sigma)}{p(p-\sigma)}\left|a_{n+p}\right||z|^{n}<1
$$

Now, from (7), we can easily see that

$$
\begin{aligned}
& \sum_{n=1}^{\infty}\left(\lambda_{2}(1+B)\left(\lambda_{1}+\alpha[p+n-1]_{q}\right)+(1-\alpha)(1+A)[p]_{q} \lambda_{1}\right)\left|a_{n+p}\right| \\
& \quad \leqq(1-\alpha)(A-B)[p]_{q} \lambda_{1}
\end{aligned}
$$

which implies that

$$
\sum_{n=1}^{\infty} \frac{\lambda_{2}(1+B)\left(\lambda_{1}+\alpha[p+n-1]_{q}\right)+(1-\alpha)(1+A)[p] \lambda_{1}}{(1-\alpha)(A-B)[p]_{q} \lambda_{1}}\left|a_{n+p}\right|<1 .
$$

The inequality in (16) will be true, if the following condition holds true:

$$
\begin{aligned}
\sum_{n=1}^{\infty} & \frac{(p+n)(n+p-\sigma)}{p(p-\sigma)}\left|a_{n+p}\right||z|^{n} \\
& <\sum_{n=1}^{\infty} \frac{\lambda_{2}(1+B)\left(\lambda_{1}+\alpha[p+n-1]_{q}\right)+(1-\alpha)(1+A)[p]_{q} \lambda_{1}}{(1-\alpha)(A-B)[p]_{q} \lambda_{1}}\left|a_{n+p}\right|,
\end{aligned}
$$

which implies that

$$
|z|^{n}<\frac{p(p-\sigma)\left(\lambda_{2}(1+B)\left(\lambda_{1}+\alpha[p+n-1]_{q}\right)+(1-\alpha)(1+A)[p]_{q} \lambda_{1}\right)}{(p+n)(n+p-\sigma)(1-\alpha)(A-B)[p]_{q} \lambda_{1}},
$$

or, equivalently, that

$$
\begin{aligned}
|z|< & \left(\frac{p(p-\sigma)\left(\lambda_{2}(1+B)\left(\lambda_{1}+\alpha[p+n-1]_{q}\right)+(1-\alpha)(1+A)[p]_{q} \lambda_{1}\right)}{(p+n)(n+p-\sigma)(1-\alpha)(A-B)[p]_{q} \lambda_{1}}\right)^{\frac{1}{n}} \\
& =r_{1} .
\end{aligned}
$$

This completes the proof of Theorem 5 .

Theorem 6. Let $f \in \mathcal{S}_{q}(p, \alpha, A, B)$. Then $f \in \mathcal{S}_{p}^{*}(\sigma)$ for $|z|<r_{2}$, where

$$
r_{2}=\left\{\inf \left(\frac{(p-\sigma)\left(\lambda_{2}(1+B)\left(\lambda_{1}+\alpha[p+n-1]_{q}\right)+(1-\alpha)(1+A)[p]_{q} \lambda_{1}\right)}{(n+p-\sigma)(1-\alpha)(A-B)[p]_{q} \lambda_{1}}\right)^{\frac{1}{n}}: n \in \mathbb{N}\right\}
$$

Proof. We know that $f \in \mathcal{S}_{p}^{*}(\alpha)$, if and only if

$$
\left|\frac{z f^{\prime}(z)-p f(z)}{z f^{\prime}(z)+(p-2 \sigma) f(z)}\right| \leqq 1 .
$$


Using (1) and upon simplification, we get

$$
\sum_{n=1}^{\infty}\left(\frac{n+p-\sigma}{p-\sigma}\right)\left|a_{n+p}\right||z|^{n}<1 .
$$

Now from (7), we can easily find that

$$
\sum_{n=1}^{\infty} \frac{\lambda_{2}(1+B)\left(\lambda_{1}+\alpha[p+n-1]_{q}\right)+(1-\alpha)(1+A)[p]_{q} \lambda_{1}}{(1-\alpha)(A-B)[p]_{q} \lambda_{1}}\left|a_{n+p}\right|<1 .
$$

For the inequality (17) to be true, it will be sufficient to show that

$$
\begin{aligned}
\sum_{n=1}^{\infty}( & \left.\frac{n+p-\sigma}{p-\sigma}\right)\left|a_{n+p}\right||z|^{n} \\
& <\sum_{n=1}^{\infty} \frac{\lambda_{2}(1+B)\left(\lambda_{1}+\alpha[p+n-1]_{q}\right)+(1-\alpha)(1+A)[p]_{q}}{(1-\alpha)(A-B)[p]_{q} \lambda_{1}}\left|a_{n+p}\right|
\end{aligned}
$$

which yields

$$
|z|^{n}<\frac{(p-\sigma)\left(\lambda_{2}(1+B)\left(\lambda_{1}+\alpha[p+n-1]_{q}\right)+(1-\alpha)(1+A)[p]_{q} \lambda_{1}\right)}{(n+p-\sigma)(1-\alpha)(A-B)[p]_{q} \lambda_{1}}
$$

and hence

$$
\begin{aligned}
& |z|<\left(\frac{(p-\sigma)\left(\lambda_{2}(1+B)\left(\lambda_{1}+\alpha[p+n-1]_{q}\right)+(1-\alpha)(1+A)[p]_{q} \lambda_{1}\right)}{(n+p-\sigma)(1-\alpha)(A-B)[p]_{q} \lambda_{1}}\right)^{\frac{1}{n}} \\
& \quad=r_{2} .
\end{aligned}
$$

We thus obtain the required result asserted by Theorem 6 .

Let the functions $f_{l}(z)(l=1,2,3, \cdots, k)$ be defined by

$$
f_{l}(z)=z+\sum_{n=1}^{\infty} a_{n, l} z^{n+p} \quad(z \in \mathbb{D}) .
$$

Now we state and prove the following results.

Theorem 7. The class $\mathcal{S}_{q}(p, \alpha, A, B)$ is closed under convex combination.

Proof. Suppose that the functions $f_{l}(z)(l=1,2)$, given by (18), belong to the class $\mathcal{S}_{q}(p, \alpha, A, B)$. Then we need to show that the function $\mathfrak{h}(z)$ given by

$$
\mathfrak{h}(z)=v f_{1}(z)+(1-v) f_{2}(z) \quad(0 \leqq \lambda \leqq 1),
$$

is also in the class $\mathcal{S}_{q}(p, \alpha, A, B)$. Indeed, for $0 \leqq v \leqq 1$, we have

$$
\mathfrak{h}(z)=z+\sum_{n=2}^{\infty}\left[v a_{n, 1}+(1-v) a_{n, 2}\right] z^{n} .
$$

Thus, if

$$
\lambda_{1}=[p-1]_{q} \quad \text { and } \quad \lambda_{2}=[p+n]_{q^{\prime}}
$$


then we have

$$
\begin{aligned}
& \sum_{n=1}^{\infty}[(1+B)\left(\lambda_{1} \lambda_{2}+\alpha \lambda_{2}[p+n-1]_{q}\right) \\
&\left.+(1-\alpha)(1+A)[p]_{q} \lambda_{1}\right]\left|v a_{n+p, 1}+(1-v) a_{n+p, 2}\right| \\
&=v \sum_{n=1}^{\infty}\left[(1+B)\left(\lambda_{1} \lambda_{2}+\alpha \lambda_{2}[p+n-1]_{q}\right)\right. \\
&\left.\quad+(1-\alpha)(1+A)[p]_{q} \lambda_{1}\right]\left|a_{n+p, 1}\right| \\
& \quad+(1-v) \sum_{n=1}^{\infty}\left[(1+B)\left(\lambda_{1} \lambda_{2}+\alpha \lambda_{2}[p+n-1]_{q}\right)\right. \\
&\left.\quad+(1-\alpha)(1+A)[p]_{q} \lambda_{1}\right]\left|a_{n+p, 2}\right| \\
& \leqq v\left[(1-\alpha)(A-B)[p]_{q} \lambda_{1}\right]+(1-v)\left[(1-\alpha)(A-B)[p]_{q} \lambda_{1}\right] \\
&=(1-\alpha)(A-B)[p]_{q} \lambda_{1} .
\end{aligned}
$$

Hence, by Theorem $1, \mathfrak{h}(z) \in \mathcal{S}_{q}(p, \alpha, A, B)$. The demonstration of Theorem 7 is thus completed.

Theorem 8. Let the L functions $f_{l}(z) \quad(l=1,2,3, \cdots, L)$, defined by (18), be in the class $\mathcal{S}_{q}(p, \alpha, A, B)$. Then the function $F(z)$, given by

$$
F(z)=\sum_{l=1}^{L} v_{l} f_{l}(z) \quad\left(\lambda_{l} \geqq 0 ; \sum_{l=1}^{L} \lambda_{l}=1\right),
$$

is also in the class $\mathcal{S}_{q}(p, \alpha, A, B)$,

Proof. The proof of Theorem 8 is fairly straightforward. We, therefore, omit the details involved.

\section{Concluding Remarks and Observations}

Applications of the $q$-calculus have been the focus point in the recent times in various branches of Mathematics and Physics mentioned [7]. In this article, we have introduced a new $q$-operator for multivalent functions. Then a new subclass of analytic and multivalent functions has been defined and studied systematically. In particular, we have investigated some of its geometric properties such as sufficient conditions, coefficient estimates, distortion Theorems, radii problems, closure-type results, and so on. The idea used in this article can easily be implemented to define several subclasses of analytic and univalent (or multivalent) functions connected with different image domains. This will open up a lot of new opportunities for research in this and related fields.

Basic (or $q$-) series and basic (or $q$-) polynomials, especially the basic (or $q$-) hypergeometric functions and basic (or $q$-) hypergeometric polynomials are applicable particularly in several diverse areas (see, for example, [46] (pp. 350-351); see also [28,29,47]). Moreover, as we remarked above and in the introductory Section 1, in Srivastava's recentlypublished survey-cum-expository review article [7], the triviality of the so-called $(p, q)$ calculus was exposed and it is also mentioned as an obviously inconsequential variation of the classical $q$-calculus, the additional parameter $p$ being redundant or superfluous (see, for details, [7] (p. 340)). Indeed one can apply Srivastava's observation and exposition in [7] to any attempt to produce the rather trivial and straightforward $(p, q)$-variations of the $q$-results which we have presented in this paper. 
Author Contributions: Conceptualization, H.M.S., M.G.K., N.K. and B.A.; methodology, H.M.S.; W.K.M. and B.K. software, B.A., N.K., M.G.K. and B.K.; validation, H.M.S., N.K. and B.K.; formal analysis, H.M.S., W.K.M., Q.H. and N.K., investigation, B.A., M.G.K. and B.K.; writing-original draft preparation, B.A., N.K., M.G.K. and B.K.; writing-review and editing, Q.H. and B.K.; visualization, H.M.S., M.G.K., N.K. and B.A; supervision, H.M.S.; project administration, Q.H.; funding acquisition, Q.H.; All authors have read and agreed to the published version of the manuscript.

Funding: This work was supported by The Key Scientific Research Project of the Colleges and Universities in Henan Province (NO. 19A110024), Natural Science Foundation of Henan Province (CN) (NO. 212300410204), (No.212300410211) and National Project Cultivation Foundation of Luoyang Normal University (No.2020-PYJJ-011).

Data Availability Statement: Not applicable.

Acknowledgments: The authors are grateful to the editor and the reviewers for their valuable comments and suggestions.

Conflicts of Interest: The authors declare that they have no conflict of interest.

\section{References}

1. Jackson, F.H. On q-definite integrals. Quart. J. Pure Appl. Math. 1910, 41, 193-203.

2. Jackson, F.H. q-Difference equations. Am. J. Math. 1910, 32, 305-314. [CrossRef]

3. Anastassiu, G.A.; Gal, S.G. Geometric and approximation properties of generalized singular integrals. J. Korean Math. Soc. 2006, 23, 425-443. [CrossRef]

4. Aral, A. On the generalized Picard and Gauss Weierstrass singular integrals. J. Comput. Anal. Appl. $2006,8,249-261$.

5. Ismail, M.E.-H.; Merkes, E.; Styer, D. A generalization of starlike functions. Complex Var. Theory Appl. 1990, 14, 77-84. [CrossRef]

6. Srivastava, H.M. Univalent functions, fractional calculus, and associated generalized hypergeometric functions. In Univalent Functions, Fractional Calculus, and Their Applications; Srivastava, H.M., Owa, S., Eds.; Halsted Press: New York, NY, USA; Ellis Horwood Limited: Chichester, UK, 1898; pp. 329-354.

7. Srivastava, H.M. Operators of basic (or $q$-) calculus and fractional $q$-calculus and their applications in geometric function theory of complex analysis. Iran. J. Sci. Technol. Trans. A Sci. 2020, 44, 327-344. [CrossRef]

8. Srivastava, H.M.; Bansal, D. Close-to-convexity of a certain family of $q$-Mittag-Leffler functions. J. Nonlinear Var. Anal. 2017, 1,61-69.

9. Kanas, S.; Răducanu, D. Some class of analytic functions related to conic domains. Math. Slovaca 2014, 64, 1183-1196. [CrossRef]

10. Mahmmod, S.; Sokół, J. New subclass of analytic functions in conical domain associated with Ruscheweyh $q$-differential operator. Results Math. 2017, 17, 1345-1357. [CrossRef]

11. Srivastava, H.M.; Tahir, M.; Khan, B.; Ahmad, Q.Z.; Khan, N. Some general classes of $q$-starlike functions associated with the Janowski functions. Symmetry 2019, 11, 292. [CrossRef]

12. Srivastava, H.M.; Tahir, M.; Khan, B.; Ahmad, Q.Z.; Khan, N. Some general families of $q$-starlike functions associated with the Janowski functions. Filomat 2019, 33, 2613-2626. [CrossRef]

13. Ahmad, B.; Khan, M.G.; Aouf, M.K.; Mashwani, W.K.; Salleh, Z.; Tang, H. Applications of a new $q$-difference operator in the Janowski-type meromorphic convex functions. J. Funct. Spaces 2021, 2021, 5534357.

14. Mahmood, S.; Ahmad, Q.Z.; Srivastava, H.M.; Khan, N.; Khan, B.; Tahir, M. A certain subclass of meromorphically $q$-starlike functions associated with the Janowski functions. J. Inequal. Appl. 2019, 2019, 88. [CrossRef]

15. Mahmood, S.; Srivastava, H.M.; Khan, N.; Ahmad, Q.Z.; Khan, B.; Ali, I. Upper bound of the third Hankel determinant for a subclass of $q$-starlike functions. Symmetry 2019, 11, 347. [CrossRef]

16. Rehman, M.S.U.; Ahmad, Q.Z.; Srivastava, H.M.; Khan, N.; Darus, M.; Khan, B. Applications of higher-order $q$-derivatives to the subclass of $q$-starlike functions associated with the Janowski functions. AIMS Math. 2021, 6, 1110-1125. [CrossRef]

17. Shi, L.; Khan, Q.; Srivastava, G.; Liu, J.-L.; Arif, M. A study of multivalent $q$-starlike functions connected with circular domain. Mathematics 2019, 7, 670. [CrossRef]

18. Shi, L.; Khan, M.G.; Ahmad, B. Some geometric properties of a family of analytic functions involving a generalized $q$-operator. Symmetry 2019, 12, 291. [CrossRef]

19. Srivastava, H.M.; Khan, B.; Khan, N.; Ahmad, Q.Z. Coefficient inequalities for $q$-starlike functions associated with the Janowski functions. Hokkaido Math. J. 2019, 48, 407-425. [CrossRef]

20. Srivastava, H.M.; Khan, B.; Khan, N.; Ahmad, Q.Z.; Tahir, M. A generalized conic domain and its applications to certain subclasses of analytic functions. Rocky Mt. J. Math. 2019, 49, 2325-2346. [CrossRef]

21. Khan, Q.; Arif, M.; Raza, M.; Srivastava, G.; Tang, H. Some applications of a new integral operator in $q$-analog for multivalent functions. Mathematics 2019, 7, 1178. [CrossRef]

22. Khan, B.; Srivastava, H.M.; Arjika, S.; Khan, S.; Khan, N.; Ahmad, Q.Z. A certain $q$-Ruscheweyh type derivative operator and its applications involving multivalent functions. Adv. Differ. Equ. 2021, 2021, 1-14. [CrossRef] 
23. Khan, B.; Liu, Z.-G.; Srivastava, H.M.; Khan, N.; Darus, M.; Tahir, M. A study of some families of multivalent $q$-starlike functions involving higher-order $q$-Derivatives. Mathematics 2020, 8, 1470. [CrossRef]

24. Khan, B.; Liu, Z.-G.; Srivastava, H.M.; Khan, N.; Tahir, M. Applications of higher-order derivatives to subclasses of multivalent q-starlike functions. Maejo Internat. J. Sci. Technol. 2021, 15, 61-72.

25. Khan, B.; Srivastava, H.M.; Khan, N.; Darus, M.; Tahir, M.; Ahmad, Q. Coefficient estimates for a subclass of analytic functions associated with a certain leaf-like domain. Mathematics 2020, 8, 1334. [CrossRef]

26. Khan, B.; Srivastava, H.M.; Tahir, M.; Darus, M.; Ahmad, Q.Z.; Khan, N. Applications of a certain integral operator to the subclasses of analytic and bi-univalent functions. AIMS Math. 2021, 6, 1024-1039. [CrossRef]

27. Khan, B.; Srivastava, H.M.; Khan, N.; Darus, M.; Ahmad, Q.Z.; Tahir, M. Applications of certain conic domains to a subclass of $q$-starlike functions associated with the Janowski functions. Symmetry 2021, 13, 574. [CrossRef]

28. Liu, Z.-G. Two q-difference equations and q-operator identities. J. Differ. Equ. Appl. 2010, 16, 1293-1307. [CrossRef]

29. Liu, Z.-G. Some operator identities and $q$-series transformation formulas. Discret. Math. 2003, 256, 119-139. [CrossRef]

30. Mahmood, S.; Raza, N.; AbuJarad, E.S.; Srivastava, G.; Srivastava, H.M.; Malik, S.N. Geometric properties of certain classes of analytic functions associated with a $q$-integral operator. Symmetry 2019, 11, 719. [CrossRef]

31. Raza, M.; Srivastava, H.M.; Arif, M.; Ahmad, K. Coefficient estimates for a certain family of analytic functions involving a q-derivative operator. Ramanujan J. 2021, 55, 53-71. [CrossRef]

32. Rehman, M.S.U.; Ahmad, Q.Z.; Srivastava, H.M.; Khan, B.; Khan, N. Partial sums of generalized q-Mittag-Leffler functions. AIMS Math. 2020, 5, 408-420. [CrossRef]

33. Srivastava, H.M.; Ahmad, Q.Z.; Khan, N.; Khan, N.; Khan, B. Hankel and Toeplitz determinants for a subclass of $q$-starlike functions associated with a general conic domain. Mathematics 2019, 7, 181. [CrossRef]

34. Srivastava, H.M.; Aouf, M.K.; Mostafa, A.O. Some properties of analytic functions associated with fractional $q$-calculus operators. Miskolc Math. Notes 2019, 20,1245-1260. [CrossRef]

35. Srivastava, H.M.; Arif, M.; Raza, M. Convolution properties of meromorphically harmonic functions defined by a generalized convolution q-derivative operator. AIMS Math. 2021, 6, 869-5885. [CrossRef]

36. Srivastava, H.M.; Khan, B.; Khan, N.; Tahir, M.; Ahmad, S.; Khan, N. Upper bound of the third Hankel determinant for a subclass of $q$-starlike functions associated with the $q$-exponential function. Bull. Sci. Math. 2021, 167, 102942. [CrossRef]

37. Srivastava, H.M.; Raza, N.; AbuJarad, E.S.A.; Srivastava, G.; AbuJarad, M.H. Fekete-Szegö inequality for classes of $(p, q)$-starlike and $(p, q)$-convex functions. Rev. Real Acad. Cienc. Exactas Fís. Natur. Ser. A Mat. (RACSAM) 2019, 113, 3563-3584. [CrossRef]

38. Yan, C.-M.; Srivastava, R.; Liu, J.-L. Properties of certain subclass of meromorphic multivalent functions associated with q-difference operator. Symmetry 2021, 13, 1035. [CrossRef]

39. Janwoski, W. Some extremal problems for certain families of analytic functions. Ann. Polon. Math. 1973, 28, 297-326. [CrossRef]

40. Dziok, J.; Murugusundaramoorthy, G.; Sokół, J. On certain class of meromorphic functions with positive coefficients. Acta Math. Sci. B 2012, 32, 1-16. [CrossRef]

41. Aldweby, H.; Darus, M. Integral operator defined by $q$-analogue of Liu-Srivastava operator. Studia Univ. Babeş-Bolyai Ser. Math. 2013, 58, 529-537.

42. Pommerenke, C. On meromorphic starlike functions. Pac. J. Math. 1963, 13, 221-235. [CrossRef]

43. Seoudy, T.M.; Aouf, M.K. Coefficient estimates of new classes of $q$-starlike and $q$-convex functions of complex order. J. Math. Inequal. 2016, 10, 135-145. [CrossRef]

44. Uralegaddi, B.A.; Somanatha, C. Certain diferential operators for meromorphic functions. Houst. J. Math. 1991, 17, $279-284$.

45. Rogosinski, W. On the coefficients of subordinate functions. Proc. Lond. Math. Soc. (Ser. 2) 1843, 48, 48-82. [CrossRef]

46. Srivastava, H.M.; Karlsson, P.W. Multiple Gaussian Hypergeometric Series; Ellis Horwood Limited: Chichester, UK, 1985.

47. Srivastava, H.M.; Seoudy, T.M.; Aouf, M.K. A generalized conic domain and its applications to certain subclasses of multivalent functions associated with the basic (or $q$-) calculus. AIMS Math. 2021, 6, 6580-6602. [CrossRef] 